\title{
Exact Self-similar Solution for Spherical Shock Wave in a Non-ideal Gas with Azimuthal Magnetic Field under Isothermal Flow Condition
}

\author{
G. Nath \\ Department of Mathematics, Motilal Nehru National Institute of Technology Allahabad, Allahabad-211004, India
}

Corresponding Author Email: gnath@mnnit.ac.in

https://doi.org/10.18280/mmc_b.882-412

Received: 2 April 2019

Accepted: 25 June 2019

\section{Keywords:}

shock wave, exact similarity solution, nonideal gas, magnetogasdynamics

\begin{abstract}
In the present paper the exact similarity solution for spherical shock wave propagation in a non-ideal gas in the case of isothermal flow with azimuthal magnetic field is studied. In the ambient medium the initial density is assumed to be constant and the magnetic field is taken to be varying according to power law. Exact self-similar solutions are obtained using Mc. Vittie method in the case of isothermal flow in non-ideal gas with magnetic field first time. To obtain the solution similarity transformations are used to transfer the system of PDE's into a system of ODE's. The product solution of Mc Vittie is used to obtain the exact self-similar solution of the problem under consideration. The effects of an increase in the values of the ratio of the gas specific heats $\gamma$ the parameter of the nonidealness of the gas and the strength of initial magnetic field $M_{A}^{-2}$ are discussed in detail. It is shown that the shock strength decreases and isothermal compressibility increases with an increase in the values of these parameters. The total energy of the shock wave increases with time. The solutions obtained show that the density, fluid velocity, magnetic field and pressure tend to zero as the point of symmetry is reached.
\end{abstract}

\section{INTRODUCTION}

Shock waves are plentiful in the Universe. Shock waves are generated when cosmic obstacles are exposed to high-speed (super-magneto-sonic) flows or, vice versa, when objects such as planets, comets, galaxies and so on pass across their gaseous environments at high speeds. Shock wave also forms due to rapid flows interaction in head-on collisions, overturn each other are stopped by their resting environments. From point of view of observation, though, astrophysics depends solely on the detection of radiation emitted, i.e. from radio waves to cosmic rays and does not allow to performed the measurements in situ on any of those objects with possibly the exclusion of shocks in the solar system and the heliosphere. Due to this limitation, only those shocks can be observed and recognized from Earth or with the help of spacecraft from Earth's orbit which radiate in one of the observable bands of the total electromagnetic spectrum. Shocks must be ionized in order to radiate, and in the majority of cases they also involve magnetic fields [1].

Pressure reduction of a moving shock wave propagating in a duct is of great significance in various applications such as safety and health, transportation (car silencers and high-speed trains), and pipe loading (the chemical industry). Shock waves propagation in tunnels from explosions creates damage and may result in loss of lives. The attenuation of shock waves in tunnels and mines, for example, is of great significance to safety point of view [2].

The self-similar problem formulations describing the adiabatic motion in gas model of stars are studied in [3-6]. In the study of astrophysical phenomena, the problem of magnetogasdynamic shock waves in interplanetary atmosphere assumes special significance. Magnetic fields permeate the universe and have vital role in a number of astrophysical situations and most likely affect all astrophysical plasmas [7, 8]. A vital role is played by magnetic fields and radiation flux in momentum and energy transport and can rapidly release energy in flares. In the field of magnetogasdynamic flows a thorough review can be seen in the work by Shang [9]. Lock and Mestel [10] studied the annular self-similar solutions in ideal magnetogasdynamics by casting the ideal magnetogasdynamic equations to a threedimensional independent system in which either the fluid pressure or magnetic pressure vanish. Also shock waves in the influence of magnetic fields have a variety of industrial and astrophysical applications for details readers are refer to the important works [7, 8, 11].

In order to obtain the similarity solution, the magnetic field in the ambient medium and shock velocity are assumed to vary as some power of the distance from the point of symmetry. Also, the initial density of the ambient medium is assumed to be constant. The radiation heat transfer equations are not used explicitly, whereas the radiation flux and other flow variables in the flow field behind the shock are evaluated from the equation of motion by the use of "product solution" of Mc Vittie [12]. Other important work related to shock wave problem using the "product solution" of Mc Vittie et al. [1218]. Nath et al. [17] have studied the shock wave propagation in a self-gravitating perfect gas with variable density in the presence of magnetic field in isothermal flow condition and they obtained the exact similarity solutions using the similarity method [12].

In extreme situation that overcome in most of the problems linked with shock waves, the supposition that the gas is ideal is no more valid. In recent years, numerous studies have been performed related to the problem of shock waves in non-ideal 
gases [19-26]

Also, Nath [18] have obtained the exact similarity solutions in the case of isothermal flow using the 'Product Solutions' of Mc Vittie [12] for shock propagation in a non-ideal gas under the influence of gravitational field for Roche model. The numerical solution for the propagation of shock waves in rotational axis symmetric perfect or non-ideal gas with the presence of magnetic field in the case of isothermal flow using similarity method was obtained in Nath and others [27, 28].

In all of the works mentioned above and known to us, the exact self-similar solution have not investigated by any of the authors using the "product solution" of Mc Vittie [12] for the propagation of shock wave in a non-ideal gas with azimuthal magnetic field in the case of isothermal flow. The motivation for the present study is the pioneering work on exact solution on shock wave propagation by Mc Vittie et al. [12, 13, 15-18]. In the present study we adopted van der Waal gas model to obtain an exact self-similar solution for the unsteady isothermal flow behind a spherical shock wave in a non-ideal gas with azimuthal magnetic field with constant initial density. The present study may be considered as the generalization of the work of Vishwakarma and Patel [13] to the case of isothermal flow and the work of Nath [18] for magnetogasdynamics without gravitational effect. The supposition of isothermal flow is physically realistic, when radiation heat transfer effects are implicitly present. The temperature behind the shock increase as the shock wave propagates and it becomes extremely high so that there is intense transfer of energy by radiation. Due to this the temperature gradient to approach zero; that is, the dependent temperature tends to become uniform behind the shock front, and the flow become isothermal [29-33]. With this supposition, we obtain the exact self-similar solution in Section 4. The effects of various parameters on the flow variables and on shock strength are discussed via Table 1 and Figures 1 (a-e) in result and discussion section 5. Also, a comparison between the solution for perfect gas and non-ideal gas is made.

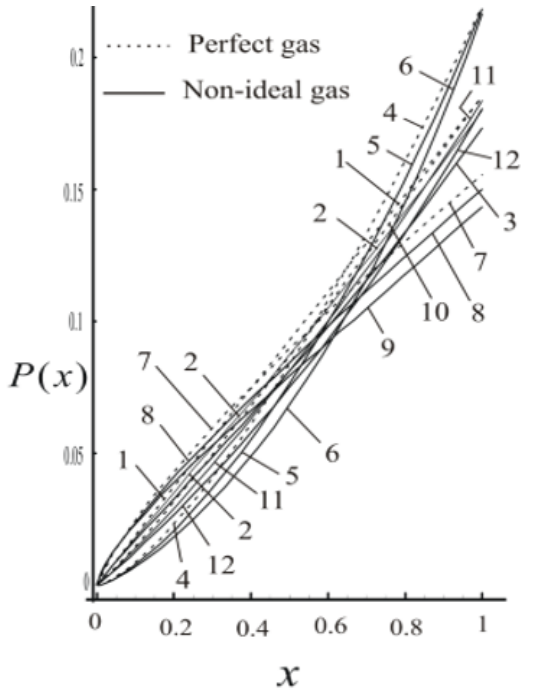

(a) pressure $P(x)$

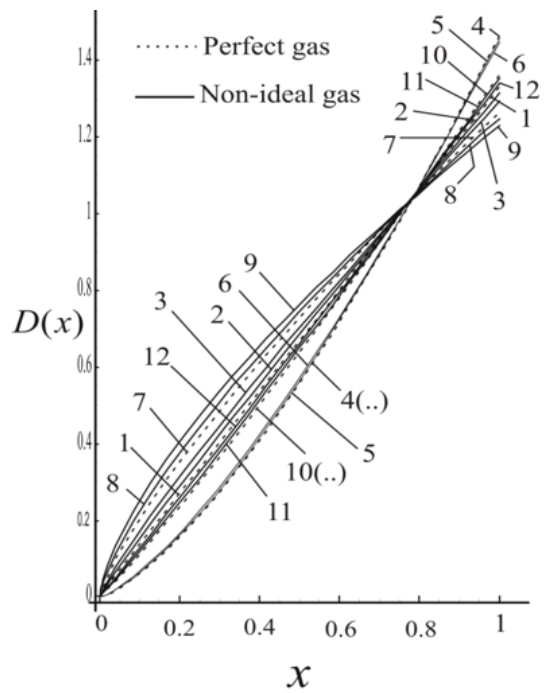

(b) density $D(x)$

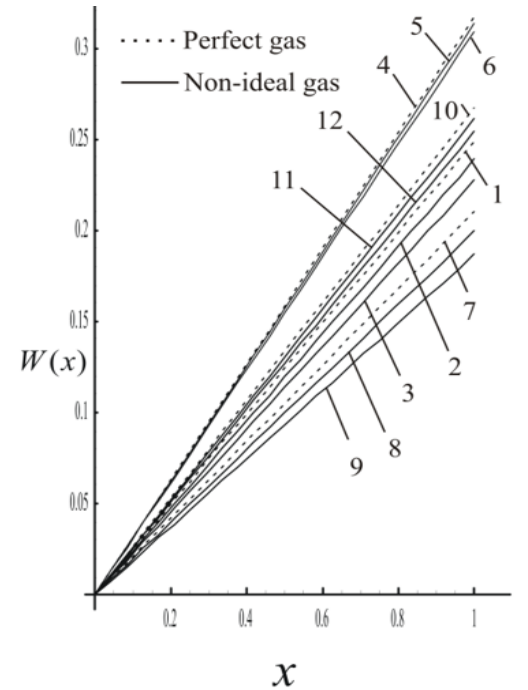

(c) fluid velocity $W(x)$

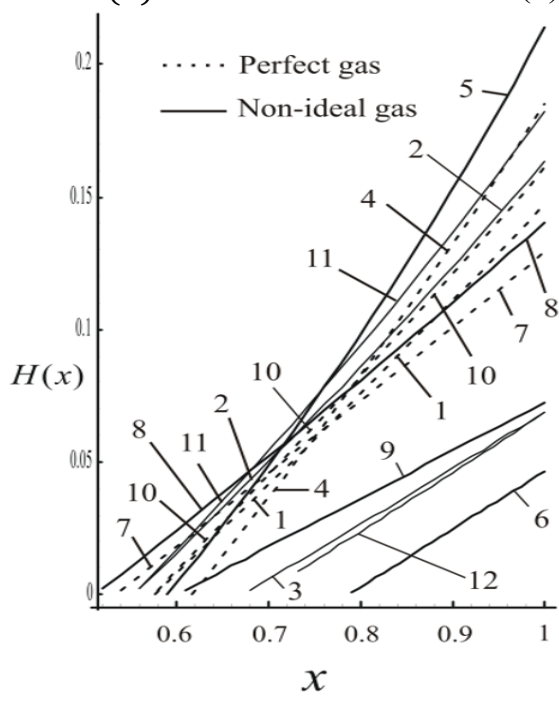

(d) azimuthal magnetic field $H(x)$

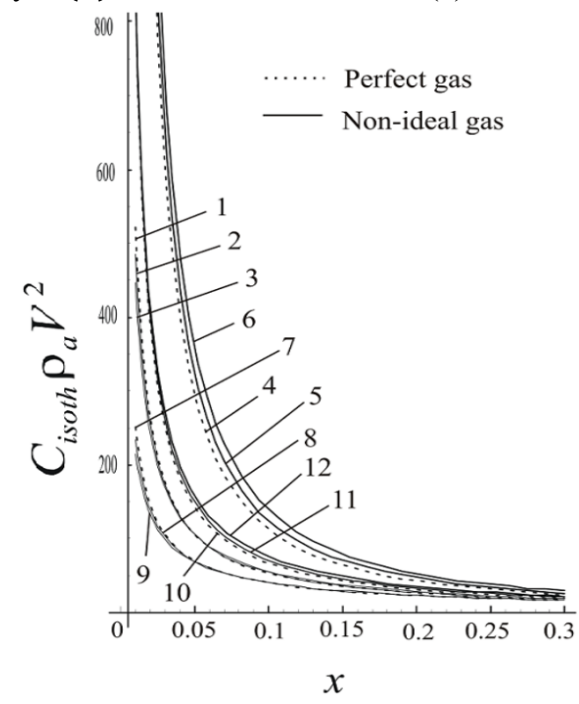

(e) isothermal compressibility $\left(C_{i s o t h}\right) \rho_{a} V^{2}$

Figure 1. Flow variables distribution in the flow field region behind the shock for $\alpha=0.5$ : Perfect gas (dashed curves) and Nonideal gas (solid curves)

Note: $1 . M_{A}^{-2}=0.2, \gamma=4 / 3, b=0 ; 2 \cdot M_{A}^{-2}=0.2, \gamma=4 / 3, \bar{b}=0.1 ; 3 \cdot M_{A}^{-2}=0.2, \gamma=\frac{4}{3}, \bar{b}=0.2 ; 4 . M_{A}^{-2}=0.2, \gamma=5 / 3, \bar{b}=0 ; 5 . M_{A}^{-2}=0.2, \gamma=5 / 3, \bar{b}=$ $0.1 ; 6 . M_{A}^{-2}=0.2, \gamma=5 / 3, \bar{b}=0.2 ; 7 . M_{A}^{-2}=0.25, \gamma=4 / 3, \bar{b}=0 ; 8 . M_{A}^{-2}=0.25, \gamma=4 / 3, \bar{b}=0.1 ; 9 . M_{A}^{-2}=0.25, \gamma=4 / 3, \bar{b}=0.2 ; 10 . M_{A}^{-2}=0.25, \gamma=$ $5 / 3, \bar{b}=0 ; 11 . M_{A}^{-2}=0.25, \gamma=5 / 3, \bar{b}=0.1 ; 12 . M_{A}^{-2}=0.25, \gamma=5 / 3, \bar{b}=0.2$ 
Table 1. The density ration across the shock front and the problem parameter values for different values of $M_{A}^{-2}, \gamma$ and $\bar{b}$

\begin{tabular}{cccccc}
\hline$M_{A}^{-2}$ & $\gamma$ & $\mathrm{M}^{2}$ & $\bar{b}$ & $\beta$ & $\lambda=\left(\frac{3}{\beta}-1\right)>2$ \\
\hline & & & 0 & 0.75158 & 2.99159 \\
& $4 / 3$ & 7.5 & 0.1 & 0.761341 & 2.94042 \\
0.2 & & & 0.2 & 0.772920 & 2.88138 \\
& & & 0 & 0.683013 & 3.39230 \\
& $5 / 3$ & 6.0 & 0.1 & 0.686539 & 3.36974 \\
& & & 0.2 & 0.690945 & 3.34188 \\
& & & 0 & 0.790161 & 2.79669 \\
0.25 & 6.3 & 0.1 & 0.800835 & 2.74609 \\
& $5 / 3$ & $5 / 3$ & 0.2 & 0.813476 & 2.68788 \\
& & & 0.1 & 0.733026 & 3.09262 \\
& & & 0.2 & 0.745832 & 3.06084 \\
\hline
\end{tabular}

\section{BASIC EQUATIONS OF MOTIONS}

The basic equations governing the one-dimensional isothermal and spherically symmetric motion of an electrically conducting non-ideal gas in the presence of azimuthal magnetic field, may be written as $(10,13,16,17,30-35)$

$$
\begin{gathered}
\frac{\partial \rho}{\partial t}+\rho \frac{\partial u}{\partial r}+u \frac{\partial \rho}{\partial r}+\frac{2 \rho u}{r}=0 \\
\frac{\partial u}{\partial t}+u \frac{\partial u}{\partial r}+\frac{1}{\rho}\left[\mu h \frac{\partial h}{\partial r}+\frac{\mu h^{2}}{2}+\frac{\partial p}{\partial r}\right]=0 \\
\frac{\partial h}{\partial t}+u \frac{\partial h}{\partial r}+h \frac{\partial u}{\partial r}+\frac{u h}{r}=0 \\
\frac{\partial T}{\partial r}=0
\end{gathered}
$$

where $t$ and $r$ are the independent time and space coordinates, $\rho$ is the density, $u$ is the fluid velocity, $h$ is the azimuthal magnetic field, $p$ is the pressure, $\mu$ is the magnetic permeability and $T$ is the temperature. The electrical conductivity of the gas is assumed to be infinite, the electrical resistivity is ignored and the diffusion term from the magnetic field equation is omitted. In the present problem the effect of rotation, viscosity and gravitation on the flow of the gas is neglected.

The supposition that the gas is ideal is no longer valid when the flow takes place at higher temperature [24]. We adopt a simple model to obtain the deviation in solution from the case of ideal gas to the non-ideal gas. We suppose that the gas follow a simplified van der Waal state equation $[21,22,26$, $28]$.

$$
p=\frac{\Gamma \rho T}{1-b \rho}, E=C_{v} T=\frac{p(1-b \rho)}{\rho(\gamma-1)}
$$

where, $\Gamma$ is the gas constant, $\gamma$ is the ratio of specific heats, $E$ is the internal energy per unit mass of the gas, $C_{v}=\frac{\Gamma}{\gamma-1}$ is the specific heat at constant volume, constant $b$ is the van der Waal excluded volume, it places a limit, $\rho \frac{1}{b_{\text {max }}}$, on the density of the gas.

For the self-similar solution [6], the shock velocity $V=\frac{d R}{d t}$ is assumed to vary as [15-17]:

$$
V^{2}=B^{2} R^{-\delta},
$$

where, $R$ is the shock radius, $B$ and $\delta$ are constants.

A spherical strong shock wave is supposed to be propagating in the undisturbed medium under the influence of azimutha magnetic field. Immediately ahead of shock front the flow variables are

$$
u_{a}=0, \rho=\rho_{a}=\text { constant }, h=h_{a}=h_{0} R^{-\alpha},
$$

where, $h_{0}$ and $\alpha$ are constants, subscript ' $a$ ' refers to the conditions immediately ahead of the shock front.

Using momentum equation (2), the initial pressure is found to be

$$
p_{a}=\frac{(1-\alpha) \mu h_{0}^{2}}{2 \alpha} R^{-2 \alpha},(0<\alpha<1)
$$

\section{BOUNDARY CONDITIONS}

The Rankine-Hugoniot shock jump conditions across the shock wave in an electrically conducting non-ideal gas are given by the principal of conservation of mass, momentum and energy, namely, [16, 24, 25, 27, 28]

$$
\begin{gathered}
\rho_{n}=\frac{\rho_{a}}{\beta}, h_{n}=\frac{h_{a}}{\beta}, u_{n}=(1-\beta) V, \\
p_{n}=\left[(1-\beta)+\frac{1}{\gamma M^{2}}+\frac{1}{2}\left(1-\frac{1}{\beta^{2}}\right) M_{A}^{-2}\right] \rho_{a} V^{2},
\end{gathered}
$$

where the subscript $n$ denotes the condition immediately behind the shock front, the density ratio $\beta(0<\beta<1)$ across the shock front is obtained by the cubic relation

$$
\begin{gathered}
(\gamma+1) \beta^{3}-\left[\frac{2}{M^{2}}+2 \bar{b}+(\gamma-1)+\gamma M_{A}^{-2}\right] \beta^{2} \\
+\beta(\bar{b}+\gamma-2)+\bar{b} M_{A}^{-2}=0
\end{gathered}
$$

where, $\left(F_{n}-F_{a}\right)$ is neglected in comparison with the product of $p_{n}$ and $V$ as the shock is strong to derive equation (10) above, $F$ is the radiation flux, $M^{2}=\frac{V^{2} \rho_{a}}{\gamma p_{a}}$ is the shock Mach number referred to the frozen speed of sound $\left(\gamma p_{a} / \rho_{a}\right)^{1 / 2}, M_{A}^{2}=\frac{V^{2} \rho_{a}}{\mu h_{a}^{2}}$ is the Alfven Mach number and $\bar{b}=b \rho_{a}[27,28,31]$.

The expressions for shock Mach number $M$ and Alfven Mach number $M_{A}$ are

$$
\begin{gathered}
M^{2}=\frac{V^{2} \rho_{a}}{\gamma p_{a}}=\frac{\rho_{a} B^{2} R^{-\delta}}{\gamma\left[\frac{(1-\alpha) \mu h_{0}^{2}}{2 \alpha}\right] R^{-2 \alpha}}, \text { and } \\
M_{A}^{2}=\frac{V^{2} \rho_{a}}{\mu h_{a}^{2}}=\frac{\rho_{a} B^{2} R^{-\delta}}{\mu h_{0}^{2} R^{-2 \alpha}} .
\end{gathered}
$$

The shock Mach number ' $M$ ' and Alfven Mach number ' $M_{A}$ ' must be constant for the flow to be self-similar. Thus the conditions for $M$ and $M_{A}$ to be constant are

$$
\delta=2 \alpha
$$

Equation (4) together with (5) gives

$$
\frac{p}{p_{n}}=\frac{\rho\left(1-b \rho_{n}\right)}{\rho_{n}(1-b \rho)}
$$


We may calculate the so-called isothermal speed of sound in non-ideal gas for a given internal volume of the gas $b$ as follows

$$
a_{i s o t h}=\left(\frac{d p}{d \rho}\right)_{T}^{\frac{1}{2}}=\left[\frac{p}{(1-b \rho) \rho}\right]^{\frac{1}{2}}
$$

where, the subscript ' $\mathrm{T}$ ' refers to the process of constant temperature.

The isothermal compressibility of non-ideal gas may be calculated as $[26]$

$$
C_{i s o t h}=\frac{1}{\rho a_{i s o t h}^{2}}
$$

\section{SIMILARITY TRANSFORMATIONS}

To obtain the self-similar solution, the unknown variables are written in the following form $[13,15]$

$$
\begin{gathered}
u=V W(x), \rho=\rho_{a} D(x), p=\rho_{a} V^{2} P(x), \\
\sqrt{\mu} h=\sqrt{\rho_{a}} V H(x),
\end{gathered}
$$

where, $x=r / R$ is the similarity variable (dimensionless variable), $W(x), D(x), P(x)$ and $H(x)$ are the function of $x$ only.

Equation (13) with the help of the equations (9) and (16) gives a relation between $D(x)$ and $P(x)$ in the form

$$
P(x)=\frac{D(x) L(\beta-\bar{b})}{(1-\bar{b} D(x))}
$$

where, $L=\left[(1-\beta)+\frac{1}{\gamma M^{2}}+\frac{1}{2}\left(1-\frac{1}{\beta^{2}}\right) M_{A}^{-2}\right]$.

Using equation (17) and similarity transformations (16), equations (1)-(3) can be transformed and simplified to

$$
\begin{gathered}
(W-x) \frac{d D}{d x}+D \frac{d W}{d x}+\frac{2 D W}{x}=0, \\
(W-x) \frac{d W}{d x}-\frac{\delta W}{2} \\
+\frac{1}{D}\left[\frac{L(\beta-\bar{b})}{(\beta-\bar{b} D)^{2}} \frac{d D}{d x}+H \frac{d H}{d x}+\frac{H^{2}}{x}\right]=0, \\
(W-x) \frac{d H}{d x}+\left(\frac{d W}{d x}-\frac{\delta}{2}\right) H+\frac{H W}{x}=0,
\end{gathered}
$$

Using the similarity transformations (16), the shock conditions (9), transformed into

$$
\begin{gathered}
W(\mathbf{1})=(\mathbf{1}-\boldsymbol{\beta}), \boldsymbol{D}(\mathbf{1})=\frac{\mathbf{1}}{\boldsymbol{\beta}}, \boldsymbol{H}(\mathbf{1})=\frac{A}{\boldsymbol{\beta}} \boldsymbol{R}^{(\delta-2 \alpha) / 2}, \\
P(1)=L,
\end{gathered}
$$

where, $A=\frac{h_{0}}{B} \sqrt{\frac{\mu}{\rho_{a}}}$.

We assume the product solution of the "progressive wave" in the form [12]

$$
\begin{gathered}
u=r \frac{a(t)}{t} \\
\rho=(\lambda+1) t^{-2 \tau} f(t) \eta^{\lambda-2},
\end{gathered}
$$

$$
\begin{gathered}
p=\tau^{2} t^{-2} b(t) f(t) \eta^{\lambda-2}, \\
h=\tau t^{-1} f^{\frac{1}{2}}(t) c(t) \eta^{\frac{\lambda}{2}}
\end{gathered}
$$

where, $\eta=r t^{-\tau}, \lambda$ and $\tau$ are constants, and $a, f, b$ and $c$ are functions of $t$ that satisfy the following equation

$$
\begin{gathered}
a(t)=\frac{\tau \lambda-t \frac{f^{\prime}(t)}{f(t)}}{\lambda+1}=\frac{\left(2-2 t \frac{c^{\prime}(t)}{c(t)}\right)}{3} \\
(\lambda-2) b(t)+\mu c^{2}(t)\left(1+\frac{\lambda}{2}\right) \eta^{2} \\
=\left(a(t)-a^{2}(t)-t a^{\prime}(t)\right) \frac{(\lambda+1) \eta^{2}}{\tau^{2}} .
\end{gathered}
$$

These equations satisfy equations (1) to (3) identically it can be easily seen. After converting this solution to a similarity one, it follows that ' $a$ ' should be a constant $a=\frac{2(1-\beta)}{(\delta+2)}$, we apply the boundary conditions (21) in (22), (23) and (25), we obtain the solution as

$$
\begin{gathered}
W(x)=(1-\beta) x, \\
D(x)=\frac{1}{\beta} x^{\lambda-2}, \\
H(x)=\frac{A}{\beta} R^{(\delta-2 \alpha) / 2} x^{\frac{\lambda}{2}}
\end{gathered}
$$

These solutions (28)-(30) satisfy differential equations (18) to (20) identically, and thus they form a solution of these differential equations (18)-(20). From equations (17) and (29)

$$
P(x)=\frac{L(\beta-\bar{b}) x^{\lambda-2}}{\left(\beta-\bar{b} x^{\lambda-2}\right)}
$$

Now, substituting equations (28)-(30) into equations (18)(19), we obtain

$$
\begin{gathered}
\lambda=\left(\frac{3}{\beta}-1\right) \\
\frac{A}{\beta} R^{(\delta-2 \alpha) / 2}= \\
\frac{(1-\beta) x^{2}(2 \beta+\delta)\left(\beta-\bar{b} x^{\lambda-2}\right)^{2}-2 \beta^{2}(\beta-\bar{b})(\lambda-2) L}{x^{2} \beta(\lambda+2)\left(\beta-\bar{b} x^{\lambda-2}\right)^{2}}
\end{gathered}
$$

The total energy $E_{\text {Total }}$ of the flow field behind the spherical shock front is given by

$$
E_{\text {Total }}=4 \pi \int_{0}^{R}\left[\frac{p(1-b \rho)}{(\gamma-1) \rho}+\frac{\mu h^{2}}{2 \rho}+\frac{1}{2} u^{2}\right] \rho r^{2} d r
$$

Using equation (17) the above equation (34) transformed into

$$
\begin{gathered}
E_{\text {Total }}=4 \pi \rho_{a} B^{2} R^{3-\delta} \int_{0}^{1}\left[\frac{P(1-\bar{b} D)}{(\gamma-1)}+\frac{1}{2}\left(H^{2}+\right.\right. \\
\left.\left.W^{2} D\right)\right] x^{2} d x
\end{gathered}
$$

This equation (35) shows that the total energy of the disturbance is not constant and varies as $R^{3-\delta}$.

Using equations (14) and (16) in (15), we obtain the expression for the isothermal compressibility as 


$$
\left(C_{\text {isoth }}\right) \rho_{a} V^{2}=\frac{(1-\bar{b} D)}{P(x)}
$$

Equations (28)-(31) provide the solution of our considered problem. The solution we have obtained is an illustration of exact similarity solution in magnetogasdynamic in a non-ideal gas with constant density in the case of isothermal flow and similar to exact solutions obtained in ordinary gas dynamics by Mc Vittie [12], in magnetogasdynamic with radiative heat flux by Vishwakarma et al. [16] in the case of adiabatic flow, and in self-gravitating perfect gas by Nath et al. [17] in the case of isothermal flow with magnetic field. The exact solutions using Mc Vittie [12], method in the case of isothermal flow in non-ideal gas with magnetic field is reported first time.

\section{RESULTS AND DISCUSSION}

From equations (11) and (12) the relation between the shock Mach number $M$ and Alfven Mach number $M_{A}$ is given by

$$
M^{2}=\frac{2 \alpha}{\gamma(1-\alpha) M_{A}^{-2}}
$$

For density and pressure to remain finite at the point of symmetry the inequality obtained from (28) and (30) i.e. $\lambda>$ 2 should hold. To obtain the solution the values of the physical parameters are taken as: $\gamma=4 / 3,5 / 3, M_{A}^{-2}=0.2,0.25 ; \alpha=$ $0.5 ; \bar{b}=0,0.1,0.2$. The obtained solutions are shown in Figures 1 and Table 1 [13, 27, 28, 35].

Figures 1 (a-d) show that the pressure $P(x)$, density $D(x)$, fluid velocity $W(x)$ andazimuthal magnetic field $H(x)$ decrease as we move from shock front to the point of symmetry. These flow variables are maximum at the shock and minimum near the point of symmetry. The isothermal compressibility increases as we move inwards from the shock front to the point of symmetry and it becomes maximum near the point of symmetry. Also, the flow variables except the isothermal compressibility $\left(C_{i s o t h}\right) \rho_{a} V^{2}$ vanish at the point of symmetry. Table 1 shows the values of the problem parameters for different values of $M_{A}^{-2}, \gamma, \bar{b}$ with $\alpha=0.5$.

Effects of an increase in the value of the strength of the initial magnetic field i.e. $M_{A}^{-2}$ from Table 1 and Figures $1(\mathrm{a}-\mathrm{e})$ are:

(1) to increase the density ratio $\beta$ across the shock front (see Table 1), i.e., to decrease the shock strength;

(2) to decrease the magnetic field $H(x)$ near shock and to increase it near point of symmetry for $\bar{b}=0.1$; whereas it increases for $\bar{b}=0.2$ in the whole flow field region behind the shock front (see Figure 1(c)).

(3) to decrease the pressure $P(x)$ and density $D(x)$ near shock and to increase these near point of symmetry (see Figure $1(\mathrm{a}, \mathrm{b}))$;

(4) to decrease the fluid velocity $W(x)$ and isothermal compressibility $\left(C_{\text {isoth }}\right) \rho_{a} V^{2}$ (see Figure $1(\mathrm{c}, \mathrm{e})$ ).

Effects of an increase in the value of the parameter of nonidealness of the gas $\bar{b}$ are:

(1) to increase the density ratio $\beta$ across the shock front (see Table 1), i.e., to decrease the shock strength;

(2) to decrease the isothermal compressibility $\left(C_{i s o t h}\right) \rho_{a} V^{2}$ for $\gamma=\frac{4}{3}$ and to increase it for $\gamma=\frac{5}{3}$ (see Figure 1(e)).

(3) to decrease the density $D(x)$ near shock and to increase it near point of symmetry (see Figure 1(b));

(4) to decrease the fluid velocity $W(x)$ (see Figure 1(c)).

(5) to decrease the magnetic field $H(x)$ and pressure $P(x)$ in general (see Figure 1(a,d)).

Effects of an increase in the value of adiabatic exponent $\gamma$ are

(1) to decrease the density ratio $\beta$ across the shock front (see Table 1), i.e., to increase the shock strength;

(2) to increase the magnetic field $H(x)$ near shock and to decrease it near point of symmetry for $\bar{b}=0.1$; whereas it decreases for $\bar{b}=0.2$ in the whole flow field region behind the shock front (see Figure 1(c)).

(3) to increase the pressure $P(x)$ and density $D(x)$ near shock and to decrease these near point of symmetry (see Figure 1(a,b))

(4) to increase the fluid velocity $W(x)$ and isothermal compressibility $\left(C_{i s o t h}\right) \rho_{a} V^{2}$ (see Figure $1(\mathrm{c}, \mathrm{e})$ ).

\section{CONCLUSION}

The exact similarity solutions for spherical shock wave propagation in a non-ideal gas with azimuthal magnetic field under isothermal flow condition have been studied. On the basis of the present work, we may draw the following conclusions:

(1) An increase in the strength of the initial magnetic field or the parameter of non-idealness $\overline{\boldsymbol{b}}$ of the gas decreases the shock strength; whereas the shock strength increases with adiabatic exponent $\gamma$.

(2) the density $D(x)$ decreases near shock and it increases near point of symmetry and the fluid velocity $W(x)$ decreases with parameter of non-idealness $\bar{b}$ of the gas. The parameter $\bar{b}$ and $M_{A}^{-2}$ have similar effect on fluid velocity $W(x)$ and density $D(x)$.

(3) The adiabatic exponent $y$ and parameter of non-idealness of the gas $\bar{b}$ have opposite effect on velocity $W(x)$ and $\operatorname{density} D(x)$.

(4) the isothermal compressibility $\left(C_{\text {isoth }}\right) \rho_{a} V^{2}$ decreases with an increase in the value of the strength of the initial magnetic field i.e. $M_{A}^{-2}$. The parameter of non-idealness $\bar{b}$ of the gas and $M_{A}^{-2}$ have similar effect on isothermal compressibility $\left(C_{i s o t h}\right) \rho_{a} V^{2}$ for $\gamma=\frac{4}{3}$ and reverse effect on it for $\gamma=\frac{5}{3}$.

(5) With an increase in $M_{A}^{-2}$ the magnetic field $H(x)$ increases near shock and it decreases near point of symmetry for $\bar{b}=0.1$; whereas it decreases for $\bar{b}=0.2$ in the whole flow field behind the shock. The pressure $P(x)$ and density $D(x)$ increases near shock and these decreases near point of symmetry with an increase in $M_{A}^{-2}$.Also, the fluid velocity $W(x)$ and isothermal compressibility $\left(C_{i s o t h}\right) \rho_{a} V^{2}$ increases with an increase in $M_{A}^{-2}$. The adiabatic exponent $\gamma$ the strength of the initial magnetic field i.e. $M_{A}^{-2}$ have similar effect on the flow variables $H(x), W(x), P(x), D(x),\left(C_{\text {isot } h}\right) \rho_{a} V^{2}$.

\section{REFERENCES}

[1] Treumann, R.A. (2009). Fundamentals of collisionless shocks for astrophysical application. 1. Non-relativistic shocks. The Astronomy and Astrophysics Review, 17(4): 
409-535. https://doi.org/10.1007/s00159-009-0024-2

[2] Mortazawy, S.M., Kontis, K., Ekaterinaris, J. (2020). Normal shock wave attenuation during propagation in ducts with grooves. Shock Waves, 30(1): 91-113. https://doi.org/10.1007/s00193-019-00916-0

[3] Zel'Dovich, Y.B., Raizer, Y.P. (2002). Physics of shock waves and high-temperature hydrodynamic phenomena. Courier Corporation.

[4] Lee, T.S., Chen, T. (1968). Hydrodynamic interplanetary shock waves. Planet. Space Sci, 16: 1483-1502.

[5] Summers, D. (1975). An idealized model of a magnetohydrodynamic spherical blast wave applied to a flare produced shock in the solar wind. Astronomy and Astrophysics, 45: 151-158.

[6] Sedov, L.I. (1993). Similarity and dimensional methods in mechanics. CRC press.

[7] Hartmann, L. (2000). Accretion processes in star formation (Vol. 32). Cambridge University Press.

[8] Balick, B., Frank, A. (2002). Shapes and shaping of planetary nebulae. Annual Review of Astronomy and Astrophysics, 40(1): 439-486.

[9] Shang, J.S. (2001). Recent research in magnetoaerodynamics. Progress in Aerospace Sciences, 37(1): 120. https://doi.org/10.1016/S0376-0421(00)00015-4

[10] Lock, R.M., Mestel, A.J. (2008). Annular self-similar solutions in ideal magnetogasdynamics. Journal of plasma physics, 74(4): 531-554. https://doi.org/10.1017/S0022377808007101

[11] Nath, G. (2019). Cylindrical shock wave generated by a moving piston in a rotational axisymmetric non-ideal gas with conductive and radiative heat-fluxes in the presence of azimuthal magnetic field. Acta Astronautica, 156: 100-112. https://doi.org/10.1016/j.actaastro.2018.10.041

[12] McVittie, G.C. (1953). Spherically symmetric solutions of the equations of gas dynamics. Proceedings of the Royal Society of London. Series A. Mathematical and Physical Sciences, 220(1142): 339-355. https://doi.org/10.1098/rspa.1953.0191

[13] Vishwakarma, J.P., Patel, N. (2015). Magnetogasdynamic cylindrical shock waves in a rotating nonideal gas with radiation heat flux. Journal of Engineering Physics and Thermophysics, 88(2): 521-530. https://doi.org/10.1007/s10891-015-1217-3

[14] Srivastava, S.K., Singh, R.K. (1983). An exact similarity solution for a spherical shock wave in a self-gravitating system. Astrophysics and Space Science, 92(2): 365-372. https://doi.org/10.1007/BF00651301

[15] Nath, G., Dutta, M., Pathak, R.P. (2017). An exact solution for the propagation of shock waves in selfgravitating medium in the presence of magnetic field and radiative heat flux. AMSE JOURNALS AMSE IIETA publication-2017 Series; Modeling B, 86(4): 907-927.

[16] Vishwakarma, J.P., Srivastava, R.C., Kumar, A. (1987). An exact similarity solution in radiation magneto-gasdynamics for the flows behind a spherical shock wave. Astrophysics and space science, 129(1): 45-52. https://doi.org/10.1007/BF00717856

[17] Nath, G., Singh, S., Srivastava, P. (2018). Exact solution for a magnetogasdynamical cylindrical shock wave in a self-gravitating rotating perfect gas with radiation heat flux and variable density. Journal of Engineering Physics and Thermophysics, 91(5): 1302-1312. https://doi.org/10.1007/s10891-018-1862-4

[18] Nath, G. (2019). Exact similarity solution for unsteady isothermal flow behind a shock wave in self-gravitating non-ideal gas. Modelling, Measurement and Control B, 88(1).

[19] Anisimov, S.I., Spiner, O.M. (1972). Motion of an almost ideal gas in the presence of a strong point explosion: PMM vol. 36, $\mathrm{n} \triangleq 5$, 1972, pp. 935-938. Journal of Applied Mathematics and Mechanics, 36(5): 883-887. https://doi.org/10.1016/0021-8928(72)90144-X

[20] Rao, M.P.R., Purohit, N.K. (1976). Self-similar piston problem in non-ideal gas. International Journal of Engineering Science, 14(1): 91-97. https://doi.org/10.1016/0020-7225(76)90059-8

[21] Wu, C.C., Roberts, P.H. (1993). Shock-wave propagation in a sonoluminescing gas bubble. Physical review letters, 70(22): 3424-3427. https://doi.org/10.1103/PhysRevLett.70.3424

[22] Roberts, P.H., Wu, C.C. (1996). Structure and stability of a spherical implosion. Physics Letters A, 213(1-2): 5964. https://doi.org/10.1016/0375-9601(96)00082-5

[23] Vishwakarma, J.P., Pandey, S.N. (2004). Magnetogasdynamic cylindrical shock waves in a nonideal gas with radiation heat flux. Modelling Meas. Control B, 73(1): 23-37.

[24] Vishwakarma, J.P., Nath, G. (2007). Similarity solutions for the flow behind an exponential shock in a non-ideal gas. Meccanica, 42(4): 331-339. https://doi.org/10.1007/s1 1012-007-9058-6

[25] Vishwakarma, J.P., Nath, G. (2009). A self-similar solution of a shock propagation in a mixture of a nonideal gas and small solid particles. Meccanica, 44(3): 239-254. https://doi.org/10.1007/s11012-008-9166-y

[26] Nath, G. (2015). Similarity solutions for unsteady flow behind an exponential shock in an axisymmetric rotating non-ideal gas. Meccanica, 50: 1701-1715.

[27] Nath, G. (2011). Magnetogasdynamic shock wave generated by a moving piston in a rotational axisymmetric isothermal flow of perfect gas with variable density. Advances in Space Research, 47(9): 1463-1471. https://doi.org/10.1016/j.asr.2010.11.032

[28] Nath, G. (2012). Propagation of a cylindrical shock wave in a rotational axisymmetric isothermal flow of a nonideal gas in magnetogasdynamics. Ain Shams Engineering Journal, 3(4): 393-401. https://doi.org/10.1016/j.asej.2012.03.009

[29] Ashraf, S., Ahmad, Z. (1975). Approximate analytic solution of a strong shock with radiation near the surface of the star. Indian Journal of Pure and Applied Mathematics, 6: 1090-1098.

[30] Korobeinikov, V.P. (1958). Unidimensional automodel motions of a conducting gas in a magnetic field. In Doklady Akad. Nauk SSSR (Vol. 121). Steklov Inst. of Math., Academy of ciences, USSR.

[31] Laumbach, D.D., Probstein, R.F. (1970). Self-Similar Strong Shocks with Radiation in a Decreasing Exponential Atmosphere. The Physics of Fluids, 13(5): 1178-1183. https://doi.org/10.1063/1.1693048

[32] Sachdev, P.L., Ashraf, S. (1971). Converging spherical and cylindrical shocks with zero temperature gradient in the rear flow field. Zeitschrift für angewandte Mathematik und Physik ZAMP, 22(6): 1095-1102. https://doi.org/10.1007/BF01590878

[33] Zhuravskaya, T.A., Levin, V.A. (1996). The propagation of converging and diverging shock waves under intense heat exchange conditions. Journal of applied 
mathematics and mechanics, 60(5): 745-752. https://doi.org/10.1016/S0021-8928(96)00094-9

[34] Rosenau, P., Frankenthal, S. (1976). Equatorial propagation of axisymmetric magnetohydrodynamic shocks. The Physics of Fluids, 19(12): 1889-1899. https://doi.org/10.1063/1.861424

\section{NOMENCLATURE}

A Abbreviation

$a(t) \quad$ Function of $\boldsymbol{t}$

$a_{i s o t h} \quad$ Isothermal sound speed

B Constant

$b \quad$ Van der Waal excluded volume

$\bar{b} \quad$ Non-idealness parameter

$b(t) \quad$ Function oft

$c(t) \quad$ Function oft

$C_{i s o t h} \quad$ Isothermal compressibility

$C_{v} \quad$ Specific heat at constant volume

$D(x) \quad$ Non-dimensional density

$E \quad$ Internal energy per unit mass

$E_{\text {Total }} \quad$ Total energy

$F \quad$ Radiation heat flux

$f(t) \quad$ Function oft

$H(x) \quad$ Non-dimensional magnetic field

$h \quad$ Azimuthal magnetic field

$h_{0} \quad$ Constant

$L \quad$ Abbreviation

$M \quad$ Shock Mach number

$M_{A}^{-2} \quad$ Alfven Mach number

$P(x) \quad$ Non-dimensional fluid pressure $p \quad$ Pressure

$R \quad$ Shock radius

$r \quad$ Independent space coordinate

$T$ Temperature of the gas

$t \quad$ Independent time coordinate

$u \quad$ Fluid velocity

$V \quad$ Shock velocity

$W(x) \quad$ Non-dimensional fluid velocity

$x \quad$ Similarity variable

\section{Greek letters}

$\rho \quad$ Fluid density

$\alpha \quad$ Magnetic field variation index

$\beta \quad$ Ratio of density across the shock

$\Gamma \quad$ Gas constant

$\gamma \quad$ Ratio of specific heats

$\delta \quad$ Constant

$\eta \quad$ Arbitrary function of $\mathrm{r}$ and $\mathrm{t}$

$\lambda \quad$ Constant

$\mu \quad$ Magnetic permeability

$\tau \quad$ Constant

\section{Subscripts}

a Immediately ahead the shock

$n \quad$ Immediately behind the shock

$T \quad$ Process of constant temperature

\section{Superscript}

Derivative with respect to $t$ 